FÉ V RIER 1971

53. ANNEE - N॰626

\title{
revue \\ internationale de la croix-rouge
}

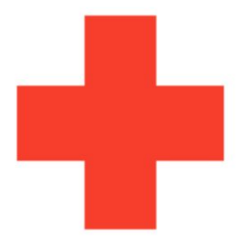

INTER ARMA CARITAS

GENEVE

COMITÉ INTERNATIONAL DE LA CROIX-ROUGE FONDÉ EN 1863 


\section{COMITÉ INTERNATIONAL DE LA CROIX-ROUGE}

MM. MARCEL A. NAVILLE, président (membre depuis 1967)

HANS BACHMANN, docteur en droit, Stadtrat de Winterthur, vice-président (1958)

JACQUES FREYMOND, docteur ès lettres, directeur de l'Institut universitaire de hautes études internationales, professeur a J'Université de Genève, vice-président (1959)

PAUL RUEGGER, ambassadeur, président du CICR de 1948 à 1955 (1948)

GUILLAUME BORDIER, ingénieur dipl. E.P.F., M.B.A. Harvard, banquier (1955)

DIETRICH SCHINDLER, docteur en droit, professeur à l'Université de Zurich (1961)

Mio MARJORIE DUVILLARD, infirmière (1961)

MM. MAX PETTTPIERRE, docteur en droit, ancien président de la Confédération suisse (1961)

ADOLPHE GRAEDEL, député au Conseil national suisse de 1951 à 1963, ancien secrétaire général de la Fédération internationale des ouvriers sur métaux (1965)

$M^{m}$ DENISE BINDSCHEDLER-ROBERT, docteur en droit, professeur a 1'Institut universitaire de hautes études internationales (1967)

MM. JACQUES F. DE ROUGEMONT, docteur en médecine (1967)

ROGER GALLOPIN, docteur en droit, ancien directeur général (1967)

JEAN PICTET, docteur en droit, président de la Commission juridique (1967)

WALDEMAR JUCKER, docteur en droit, secrétaire de l'Union syndicale suisse (1967)

HARALD HUBER, docteur en droit, juge fédéral (1969)

VICTOR H. UMBRICHT, docteur en droit, administrateur (1970)

Membres honoraires: M. JACQUES CHENEVIERE, vice-president d'honneur; M"1e LUCIE ODIER, vice-presidente d'honneur; MM. MARTIN BODMER, CARL J. BURCKHARDT, PAUL CARRY, $M^{\text {m }}$ MARGUERITE GAUTIER-VAN BERCHEM, MM. SAMUEL A. GONARD, EDOUARD DE HALLER, PAUL LOGOZ, HANS MEULI, RODOLFO OLGIATI, FRÉdÉRIC SIORDET, ALFREDO VANNOTTI, ADOLF VISCHER.

Direction: MM. JEAN-LOUIS LE FORT, secrétaire général;

RAYMOND COURVOISIER, assistant spécial du président et directeur du département des opérations;

CLAUDE PILLOUD, directeur du departement des principes et du droit. 


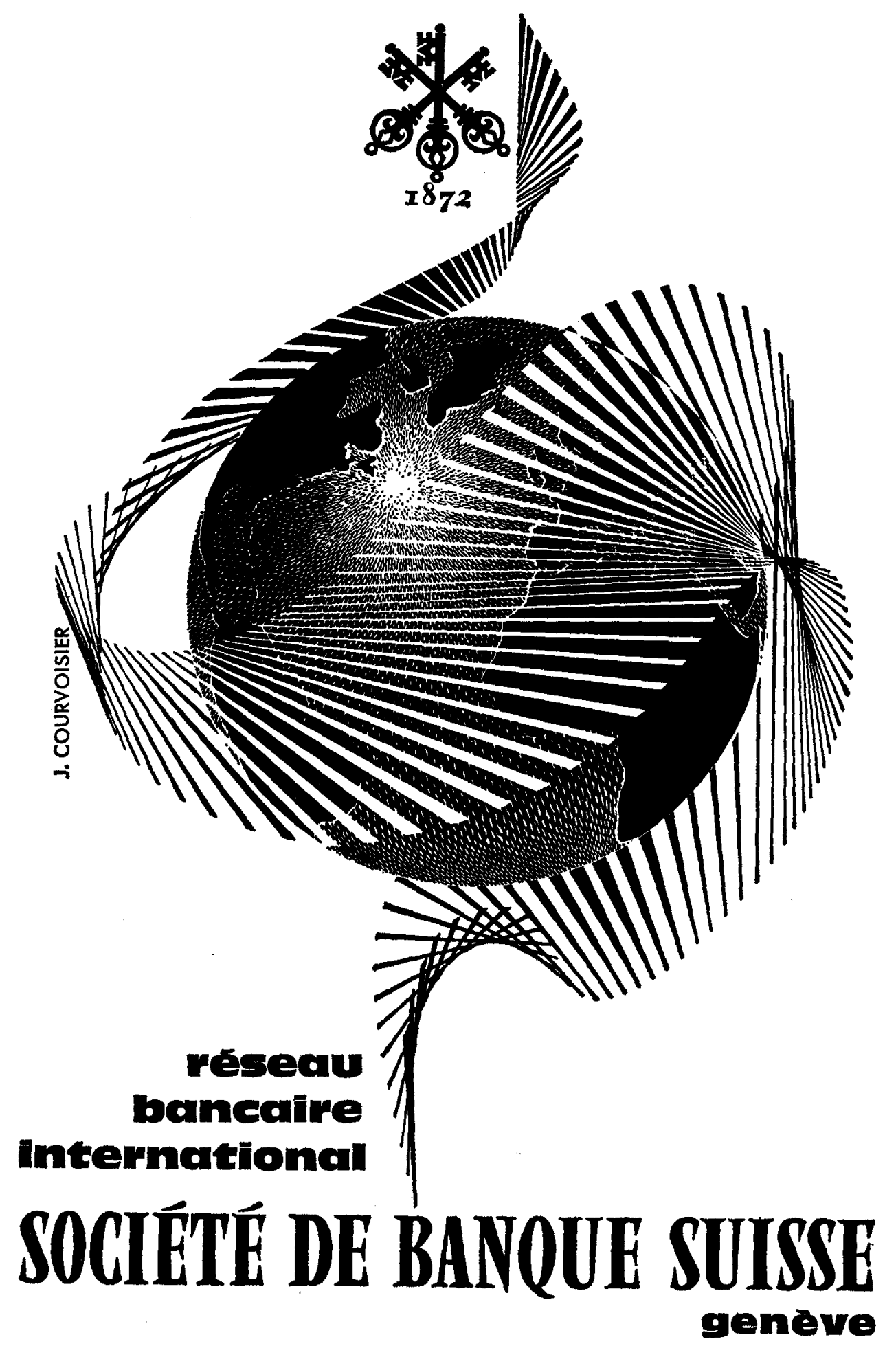




\section{SOCIETE FIDUCIAIRE ROMANDE OFOR S.A.}

Place Saint-Gervais 1, Genève - Tél. 317050

$\downarrow$ Expertises, revisions et organisations comptables

Domiciliation et administration de sociétés

^ Assainissements et liquidations

४ Interventions et conseils en matière fiscale

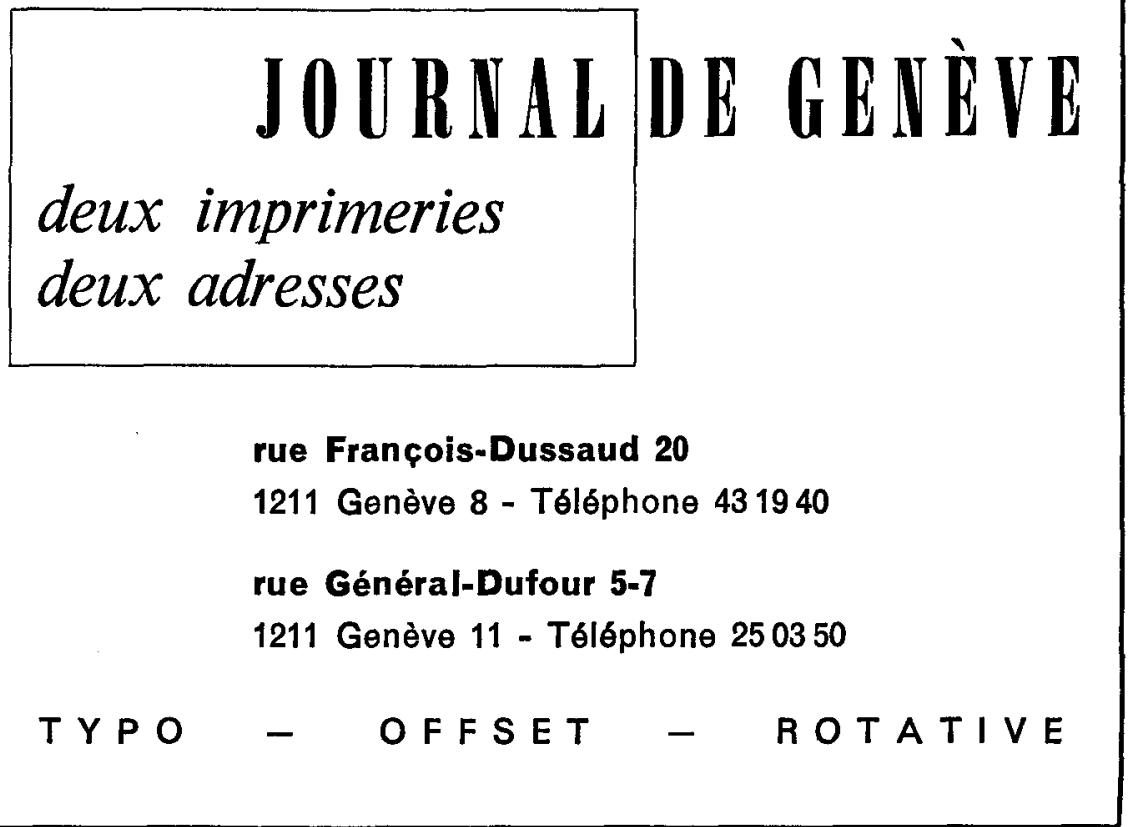




\section{REVUE INTERNATIONALE DE LA CROIX-ROUGE}

FEVRIER 1971 - № 626

\section{SOMMAIRE}

COMITÉ INTERNATIONAL DE LA CROIX-ROUGE

\section{DANS LE MONDE DE LA CROIX-ROUGE}

\section{FAITS ET DOCUMENTS}

LIVRES ET REVUES
Max Petitpierre : Actualité du Comité international de la Croix-Rouge . . . . . . . . . .

Activités extérieures:

Le président du CICR en Amérique latine - Au Proche-Orient - En République arabe du Yémen - En République khmère - En République du Vietnam - En Afrique australe .

Que fait le Service du personnel sanitaire du CICR ? . . . . . . . . . . . 102

IX ${ }^{\mathrm{e}}$ Conférence interaméricaine de la Croix-Rouge 104 Institut régional de formation pour l'Afrique orientale . . . . . . . . . . . 110

Halti . . . . . . . . . . . . . . . 111

Inde . . . . . . . . . . . 112

Liban . . . . . . . . . . . . . . . 114

Respect des droits de l'homme en période de conflit armé (II) . . . . . . . . . . . . . 115

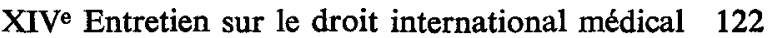




\section{INTERNATIONAL REVIEW \\ OF THE RED CROSS}

\section{SUPPLEMENTS \\ DE LA. REVUE}

EN LANGUE ESPAGNOLE

EN LANGUE ALLEMANDE
REVUE INTERNATIONALE DE LA CROIX-ROUGE
Une édition en langue anglaise paraît chaque mois. Elle est en principe identique à l'édition française, et peut être obtenue aux mêmes conditions.

C. Pilloud: Protección de los periodistas en misión peligrosa en las zonas de conflicto armado - El Presidente del CICR en América Latina - IX Conferencia Interamericana de la Cruz Roja - Respeto de los derechos humanos en período de conflicto armado.

P. Laroque: Erziehung zur Solidarität.

Elle est publiée chaque mois par le Comité international de la Croix-Rouge.

7, avenue de la Paix, 1211 Genève 1 (Suisse) - Compte de chèques postaux $12-1767$.

Abonnement un an: Fr. 25,-; le numéro: Fr. 2,50.

Pour les pays suivants: Algérie, Allemagne, Autriche, Belgique, Danenaark, Finlande, France, Italie, Luxembourg, Maroc, Norvège, Pays-Bas, Portugal, Sarre, Suede, Tunisie, Vatican, on peut s'abonner aupress des bureaux de poste.

RÉDACTION: J.-G. LOSSIER

Seuls les textes signés par le Comité international de la CroixRouge engagent la responsabilité de celui-ci. 